\title{
Application of the Updated Framingham Risk Score to Japanese Men
}

\author{
Machi SUKA, Hiroki SUGIMORI, and Katsumi YOSHIDA
}

\begin{abstract}
Few tools for risk assessment of coronary heart disease (CHD) have yet been made available in Japan. This study aims to examine the validity of the updated Framingham risk score as applied to a Japanese male population. Using the annual health examination database of a Japanese company, we followed-up 5,611 male subjects, aged 30 to 59 years, who had initially recorded neither history of cardiovascular disease nor electrocardiographical ischemic changes, in order to observe the occurrence of CHD over a period of 5 to 7 years. The total score calculated by the Framingham risk score sheet (the Framingham point score) was used as an indicator of CHD risk for the subject individually. The mean of the Framingham point score for $80 \mathrm{CHD}$ cases was significantly higher than that for 5,531 non-CHD cases. The incidence of CHD gradually increased with the Framingham point score. In the receiver operating characteristic analysis, the area under the curve reached 0.71 . At 6 points, the curve came closest to the upper left-hand corner, with a specificity of 0.74 and sensitivity of 0.59 . On the other hand, multivariable-adjusted relative risks associated with old age, high blood pressure, low HDL cholesterol and smoking in the Japanese male population were different from those in the Framingham population. Despite the low incidence of $\mathrm{CHD}$, the updated Framingham risk score could provide a reasonable rank ordering of CHD risk and could identify Japanese men (and possible other individuals) at high risk for CHD with considerable accuracy. However, further study of Japanese populations may be required to reappraise several coefficients of risk factor in the risk scoring model.
\end{abstract}

(Hypertens Res 2001; 24: 685-689)

Key Words: follow-up studies, coronary heart diseases, risk assessment

\section{Introduction}

The lifestyles of the Japanese have been westernized at a remarkable pace over the last century. Although mortality from coronary heart disease (CHD) in Japan remains lower than that in Western countries, there will possibly be an upward trend within a few decades $(1-3)$. In order to promote effective primary prevention against CHD, it is important to identify high-risk individuals who require intensive interventions to minimize the CHD risk. However, few tools are currently available in Japan for the risk assessment of CHD.

Recently, Wilson et al. (4) presented an updated version of the Framingham risk score that provides the probabilities of contracting CHD over the next 10 years (10-year CHD risk percentage) in individuals free of CHD. They incorporated only six of the major cardiovascular risk factors-age, smoking, diabetes, blood pressure, total cholesterol and HDL cholesterol-into the risk scoring model, which they adapted to a system of simple score sheets. By using categorical risk factors, the scoring procedures have also been simplified (5, 6). Moreover, in the updated Framingham risk score, the blood pressure and cholesterol categories are consistent with the categories given by the Joint National Committee (JNCV) and National Cholesterol Education Program, Adult Treatment Panel (NCEP ATP II), respectively.

The Framingham risk score has been widely accepted in Western countries because of its well-established validity

From the Department of Preventive Medicine, St. Marianna University School of Medicine, Kanagawa, Japan.

Address for Reprints: Machi Suka, M.D., Department of Preventive Medicine, St. Marianna University School of Medicine, 2-16-1 Sugao, Miyamac-

ku, Kawasaki 216-8511, Japan. E-mail: suka@marianna-u.ac.jp

Received July 23, 2001: Accepted in revised form August 27, 2001. 
Table 1. Baseline Characteristics in Japanese Male Workers Study

\begin{tabular}{lc}
\hline Age (y.o.) & $44.7 \pm 4.5$ \\
Smoking (\%) & 59.8 \\
Diabetes (\%) & 8.5 \\
Systolic blood pressure (mmHg) & $129.0 \pm 17.1$ \\
Diastolic blood pressure (mmHg) & $80.3 \pm 10.6$ \\
Total cholesterol (mmol/l) & $5.1 \pm 0.9$ \\
HDL cholesterol (mmol/l) & $1.3 \pm 0.4$ \\
\hline
\end{tabular}

Values are given as the mean \pm SD or as a percentage.

$(7,8)$. It is uncertain whether it will be equally applicable to the Japanese (9), since relationships between CHD and its risk factors are heterogeneous by ethnicity $(10)$. In this study, we focused on the updated Framingham risk score presented by Wilson et al. (4) and attempted to determine its extensibility to a Japanese male population.

\section{Methods}

A longitudinal large cohort database was accumulated from an employee health management center in a Japanese company. The database covered all annual health examinations between 1991 and 1998. We recruited 5,931 eligible male participants in the annual health examinations between 1991 and 1993 who were aged 30 to 59 years and for whom baseline measurements of the six risk factors (age, smoking, diabetes, blood pressure, total cholesterol and HDL cholesterol) were available. Those who had reported a history of CHD or stroke $(n=59)$ and those who had shown more than one of the ischemic changes (abnormal Q wave, ST-segment depression and $\mathrm{T}$-wave inversion) in a 12-lead electrocardiographic examination ( $n=148$, including those who had reported a history of CHD or stroke) were excluded. Out of the remaining 5,777 subjects, 5,611 subjects $(97 \%)$ who had received health examinations repeatedly over a 5 to 7 year period were included.

The participants in the annual health examinations were asked to complete a structured and comprehensive questionnaire about their health conditions and lifestyles. Systolic and diastolic blood pressures were measured after $5 \mathrm{~min}$ of rest in a sitting position by well-trained nurses. A 12-lead electrocardiograph was recorded, and blood samples (fasting) were taken for measurements of biochemical and hematological variables. The above processes were conducted according to the guidelines established by the employee health management center. Table 1 shows baseline characteristics of the study subjects. Smoking was defined as regular smoking in the past 5 years, which included current smokers $(92 \%)$ and those who had quit smoking for less than 5 years $(8 \%)$ at baseline. Diabetes was defined as the use of antidiabetic medications or fasting plasma glucose exceeding 7.8 $\mathrm{mmol} / \mathrm{l}$.
Information on the occurrence of CHD had been collected through both the questionnaires and the physicians' interviews in the annual health examinations between 1996 and 1998. Those who had reported the occurrence of CHD (either angina pectoris, myocardial infarction or coronary insufficiency diagnosed and treated in a hospital) for 5 to 7 years were recognized as CHD cases.

CHD risk for each subject was assessed based on the updated Framingham risk score presented by Wilson et al. (4). Figure 1 shows the Framingham risk score sheet for men (with total cholesterol). Using this score sheet, the six risk factors at baseline were converted into the respective point scores. The sum of these point scores (the Framingham point score) was used as an indicator of CHD risk for the subject individually. Categorizations of blood pressure, total cholesterol and HDL cholesterol were made without regard to the use of antihypertensive medication and antilipemic medication, respectively.

\section{Statistical Analyses}

Statistical analyses were performed using version 6.12 software from Statistical Analysis Systems (Cary, USA). The means of Framingham point scores for CHD cases and nonCHD cases were compared by Student's $t$-test. The incidence of $\mathrm{CHD}$ was calculated using the Framingham point score. After plotting a receiver operating characteristic (ROC) curve, the discriminatory performance of the risk score was measured by the area under the curve (AUC) (11); an AUC of greater than 0.5 indicates that the risk score can correctly identify CHD cases better than chance. We determined the point closest to the upper left-hand corner on the curve, which is recognized as a rational critical cut-off (7).

Moreover, multivariable-adjusted relative risks (RRs) and their corresponding $95 \%$ confidence intervals for incidence of CHD were calculated using a multiple logistic regression model, which simultaneously included the six risk factors categorized according to the definitions presented by Wilson et al (4). The RRs of the six risk factors in the Japanese population were compared with those in the Framingham population. (4).

\section{Results}

Eighty CHD cases were successfully observed for a period of 5 to 7 years, which accounts for $1.4 \%$ of the study subjects. As shown in Fig. 2, the percent distribution of Framingham point scores in the CHD cases appeared to be shifted to the right (toward the region of higher point scores) compared with that in the non-CHD cases. The mean \pm SD of the Framingham point scores for the CHD cases was significantly higher than that for the non-CHD cases $(5.8 \pm 2.7 \mathrm{vs}$. $3.7 \pm 2.7 ; p<0.001$ ). As shown in Fig. 3, the incidence of CHD gradually increased with the Framingham point score. The rate in the strata of more than 10 points reached $5.5 \%$, 


\begin{tabular}{|l|r|}
\hline Age, y.o & pts \\
\hline $30-34$ & -1 \\
\hline $35-39$ & 0 \\
\hline $40-44$ & 1 \\
\hline $45-49$ & 2 \\
\hline $50-54$ & 3 \\
\hline $55-59$ & 4 \\
\hline $60-64$ & 5 \\
\hline $65-69$ & 6 \\
\hline $70-74$ & 7 \\
\hline
\end{tabular}

\begin{tabular}{|l|r|}
\hline Smoking & pts \\
\hline No & 0 \\
\hline Yes & 2 \\
\hline Diabetes & pts \\
\hline No & 0 \\
\hline Yes & 2 \\
\hline
\end{tabular}

\begin{tabular}{|l|r|}
\hline $\begin{array}{l}\text { Total cholesterol, } \\
\mathrm{mmol} / \mathrm{I}\end{array}$ & pts \\
\hline$<4.14$ & -3 \\
\hline $4.15-5.17$ & 0 \\
\hline $5.18-6.21$ & 1 \\
\hline $6.22-7.24$ & 2 \\
\hline$\geqq 7.25$ & 3 \\
\hline
\end{tabular}

\begin{tabular}{|l|r|}
\hline $\begin{array}{l}\text { HDL cholesterol, } \\
\mathrm{mmol} / \mathrm{I}\end{array}$ & pts \\
\hline$<0.90$ & 2 \\
\hline $0.91-1.16$ & 1 \\
\hline $1.17-1.55$ & 0 \\
\hline$\geqq 1.56$ & -1 \\
\hline
\end{tabular}

\begin{tabular}{|l|l|l|l|l|l|}
\hline \multicolumn{2}{|l|}{$\begin{array}{l}\text { Blood pressure, } \\
\mathrm{mmHg}\end{array}$} & \multicolumn{4}{|c|}{ diastolic } \\
\cline { 3 - 6 } & $<85$ & $85-89$ & $90-99$ & $\geqq 100$ \\
\hline \multirow{4}{*}{ systolic } & $<130$ & 0 pts & & & \\
\cline { 2 - 5 } & $130-139$ & \multicolumn{2}{|c|}{$1 \mathrm{pts}$} & & \\
\cline { 2 - 5 } & $140-159$ & \multicolumn{3}{|c|}{$2 \mathrm{pts}$} \\
\cline { 2 - 5 } & $\geqq 160$ & \multicolumn{3}{|c|}{3 pts } \\
\hline
\end{tabular}

$\underset{\substack{\text { Framingham } \\ \text { point score }}}{\text { The }}=\underset{\text { pts }}{\text { Age }}+\underset{\text { pts }}{\text { Smoking }}+\underset{\text { pts }}{\text { Diabetes }}+\underset{\text { pressure }}{\text { pts }}+\underset{\substack{\text { plood } \\ \text { cholesterol }}}{\text { Total }}+\underset{\substack{\text { pts } \\ \text { cholesterol }}}{\text { HDL }}$

Fig. 1. Framingham risk score sheet for men (with total cholesterol). Pts indicates points. Six risk factors are converted into the respective point scores. The sum of these point scores (the Framingham point score) is used as an indicator of coronary heart disease risk for the subject individually. Categorizations of blood pressure, total cholesterol and HDL cholesterol are made without regard to the use of antihypertensive medication and antilipemic medication, respectively. Refer to Fig. 3 in Wilson et al. (4).

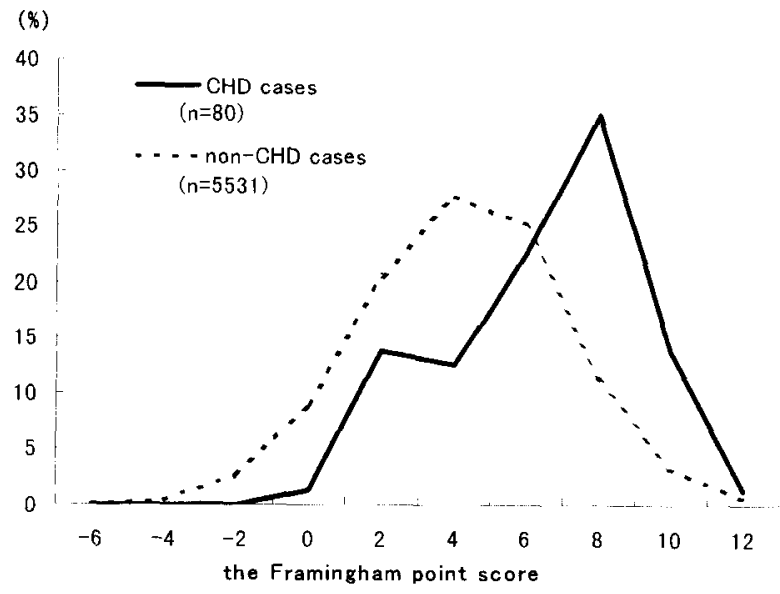

Fig. 2. Percent distribution of the Framingham point score in Japanese male workers study: $C H D$ cases $(\mathrm{n}=80)$ vs. non- $C H D$ cases $(\mathrm{n}=5,531)$. The Framingham point score was calculated by the Framingham risk score sheet for men (with total cholesterol) shown in Fig. 1 .

whereas no CHD cases were observed among those who possessed minus points.

The ROC analysis indicated that Framingham point brought out more than $70 \%$ of discriminatory performance; AUC reached 0.71 . At 6 points, the ROC curve came closest to the upper left-hand corner, with a specificity of 0.74 and sensitivity of 0.59 .
(\%)

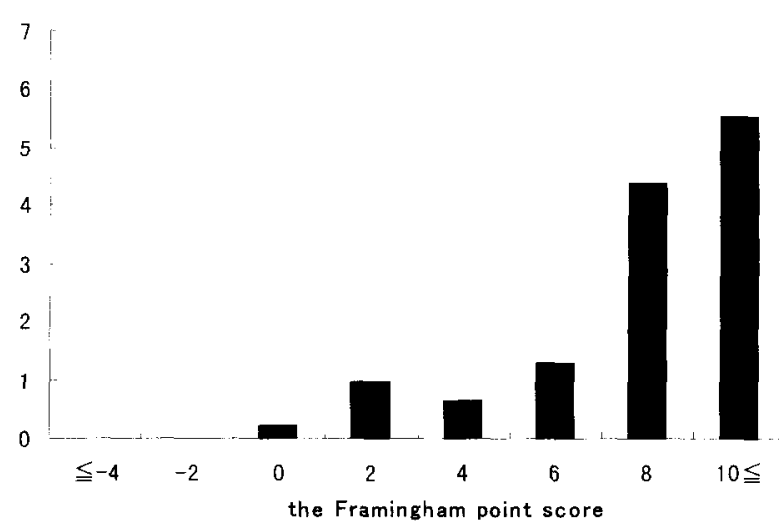

Fig. 3. Incidence of coronary heart disease by the Framingham point score in Japanese male workers study. The Framingham point score was calculated by the Framingham risk score sheet for men (with total cholesterol) shown in Fig. I.

Table 2 shows the RRs of the six risk factors in the Japanese male population. The risk for incidence of CHD increased with increase in age, blood pressure and total cholesterol and with decrease in HDL cholesterol $(p<0.05$ for all); whereas no statistically significant association was observed for smoking or diabetes. Compared with the Framingham population (4), the Japanese male population had consider- 
Table 2. Comparisons of Multivariable-Adjusted Relative Risks (95\% Confidence Intervals) for Incidence of Coronary Heart Disease: Japanese Male Workers Study vs. Framingham Study

\begin{tabular}{|c|c|c|c|c|}
\hline & \multicolumn{2}{|c|}{ Japanese male workers study $^{\dagger}$} & \multicolumn{2}{|c|}{ Framingham study } \\
\hline & Relative risk & $(95 \% \mathrm{CI})$ & Relative risk & $(95 \% \mathrm{CI})$ \\
\hline Age (y.o.) & 1.12 & $(1.06-1.18)$ & 1.05 & $(1.04-1.06)$ \\
\hline Smoking & 0.88 & $(0.56-1.40)$ & 1.68 & $(1.37-2.06)$ \\
\hline Diabetes & 1.73 & $(0.94-3.01)$ & 1.50 & $(1.06-2.13)$ \\
\hline \multicolumn{5}{|l|}{ Blood pressure } \\
\hline Normal & 1.00 & (referent) & 1.00 & (referent) \\
\hline High-normal & 1.18 & $(0.59-2.29)$ & 1.31 & $(0.98-1.76)$ \\
\hline Hypertension stage 1 & 2.69 & $(1.56-4.69)$ & 1.67 & $(1.28-2.18)$ \\
\hline Hypertension stages $2-4$ & 2.78 & $(1.24-5.82)$ & 1.84 & $(1.37-2.49)$ \\
\hline \multicolumn{5}{|l|}{ Total cholesterol (mmol/l) } \\
\hline$<5.17$ & 1.00 & (referent) & 1.00 & (referent) \\
\hline $5.18-6.21$ & 1.50 & $(0.92-2.46)$ & 1.31 & $(1.01-1.68)$ \\
\hline $6.22 \leqq$ & 2.11 & $(1.07-3.94)$ & 1.90 & $(1.47-2.47)$ \\
\hline \multicolumn{5}{|l|}{ HDL cholesterol (mmol/l) } \\
\hline $1.56 \leqq$ & 0.76 & $(0.41-1.33)$ & 0.56 & $(0.37-0.83)$ \\
\hline $0.91-1.55$ & 1.00 & (referent) & 1.00 & (referent) \\
\hline$<0.90$ & 2.95 & $(1.60-5.20)$ & 1.47 & $(1.16-1.86)$ \\
\hline
\end{tabular}

Each multivariate model simultaneously incorporated all variables listed in the table. Categorizations of blood pressure, total cholesterol and HDL cholesterol were made without regard to the use of antihypertensive medication and antilipemic medication, respectively. ${ }^{\dagger}$ Multiple logistic regression analysis was performed in 5,611 Japanese male workers. " Refer to Table 4 in Wilson et al. (4).

ably higher RRs associated with old age, high blood pressure and low HDL cholesterol, and considerably lower RRs associated with smoking.

\section{Discussion}

CHD risk depends on a number of cardiovascular risk factors, such as smoking, glucose intolerance, hypertension and hyperlipidemia. Considering the interactions of cardiovascular risk factors, a comprehensive indicator of CHD risk should be developed as a tool for risk assessment of CHD. In this study, the updated Framingham risk score, a famous tool for risk assessment of CHD (12), was examined to validate its application to a Japanese male population.

This study shows an incidence of CHD equivalent to those reported in other Japanese studies (13-15), but this incidence is much lower than those in the United States (7) or Western Europe $(16,17)$. Despite this low incidence of CHD, the updated Framingham risk score was able to provide a reasonable rank ordering of CHD risk; the incidence of CHD gradually increased with the Framingham point score (Fig. 3). Moreover, the ROC analysis indicated that the risk score could identify high-risk individuals for $\mathrm{CHD}$ with considerable accuracy. According to AUC, the discriminatory performance of the risk score in the Japanese male population was equivalent to that in the Framingham populations (0.71 vs. 0.73) (4). These results suggest that the updated Framingham risk score might be useful in risk assessment of CHD in Japanese men.
The pathway from risk assessment to risk reduction consists of three steps: 1) collection of an individual's data; 2) interpretation of the data with respect to risk assessment; and 3) intervention on the basis of the risk assessment (9). In Japan, health examinations are performed regularly as a public health service (step 1). However, Japanese clinicians have few tools available to them for risk assessment of CHD. Accordingly, in Japan, it is hardly adequate to identify high-risk individuals for $\mathrm{CHD}$ (step 2) and then take an interventional approach to minimize their CHD risks (step 3). For a preventive strategy against CHD, Japanese clinicians have need of an appropriate screening tool for the Japanese.

It is entirely possible that the updated Framingham risk score will show an acceptable discriminatory performance in Japanese men. Moreover, the scoring procedures are simple enough to be readily utilized by clinicians. To act as a screening tool, the risk score must provide a critical cut-off defining high-risk individuals who should receive vigorous preventive management against the occurrence of CHD. In the ROC analysis, a Framingham point score of 6 was recognized as a rational critical cut-off. At the same time, since $26.4 \%$ of the present cohort showed scores higher than 6 , this critical cut-off might not be suitable for practical use. Alternatively, a Framingham point score of 8 might be recommended as a reasonable critical cut-off for the following reasons: 1) at scores higher than 8 , the incidence of CHD sharply increased (Fig. 3); 2) a specificity of greater than 0.9 was obtained at scores above 8 which may adequately avoid false-negative results (ignorance of high-risk individuals); 
and 3 ) $7.8 \%$ of the study subjects were defined as high-risk individuals. However, the bias due to the so-called "healthy worker effect" in this study should be considered in the discussion about critical cut-off. It is a well-known fact that a working population is likely to include a smaller number of high-risk individuals than a general population. Thus, the Framingham point scores of a general population may rank higher than those of a working population. Further study of other Japanese populations may be required to identify a reasonable critical cut-off for the updated Framingham risk score, which may contribute to primary prevention against CHD as an appropriate screening tool for the Japanese.

This study demonstrated the validity of the updated Framingham risk score for application to a Japanese male population. At the same time, the RRs associated with old age, high blood pressure, low HDL cholesterol and smoking in the Japanese male population were different from those in the Framingham population (Table 2). This result was consistent with the findings of the Seven Countries Study, a famous multilateral study concerning CHD: in comparison with cohorts in other participating countries, two cohorts in Japan showed that systolic blood pressure had a strong influence, and serum cholesterol had a weak influence, on CHD (10, 18,19 ). Further studies on Japanese populations may be required to reappraise several coefficients of risk factor in the risk scoring model, and possibly to reform the risk score to accommodate Japanese-specific characteristics.

\section{References}

1. Health and Welfare Statistics Association: Trend of National Health. Tokyo, Health and Welfare Statistics Assoc., 1998 (in Japanese).

2. Health Service Bureau, Ministry of Health and Welfare: Report of the 4th Cardiovascular Disease Basic Survey. Tokyo, Ministry of Health and Wclfare, 1993 (in Japanese).

3. Fukiyama K, Kimura Y, Wakugami K, Muratani H: Incidence and long-term prognosis of initial stroke and acute myocardial infaretion in Okinawa, Japan. Hypertens Res 2000; 23: 127-135.

4. Wilson PWF, D'Agostino RB, Levy D, Belanger AM, Silbershatz. H, Kannel WB: Prediction of coronary heart disease using risk factor categories. Circulation 1998; 97 : 1837-1847.

5. Kannel WB, McGee D, Gordon T: A general cardiovascular risk profile: the Framingham study. Am J Cardiol 1976; 38: $46-51$.
6. Anderson KM, Odell PM, Wilson PWF, Kannel WB: Cardiovascular discase risk profiles. Am Heart J 1991; 121: 293-298.

7. Liao Y, McGee DL, Cooper RS, Sutkowski MB: How generalizable are coronary risk prediction models? Comparison of Framingham and two national cohorts. Am Heart J 1999; 137: 837-845.

8. Knuiman MW, Vu HT: Prediction of coronary heart disease mortality in Busselton Western Australia: an evaluation of the Framingham, national health cpidemiologic follow up study, and WHO ERICA risk scores. J Epidemiol Community Health 1997; 51: 515-519.

9. Greenland P, Grunndy S, Pasternak RC, Lenfant C: Problems on the pathway from risk assessment to risk reduction. Circulation 1998; 97: 1761-1762.

10. Menoti A, Keys A, Blackburn H, et al: Comparison of multivariate predictive power of major risk factors for coronary heart diseases in different countries: results from eight nations of the seven countries study, 25-year follow-up. $J$ Cardiovasc Risk 1996; 3: 69-75.

11. Erdreich LS, Lee ET: Use of relative operating characteristic analysis in epidemiology: a method for dealing with subjective judgment. Am J Epidemiol 1981; 114: 649-662.

12. Grundy SM, Pasternak R, Greenland P, Smith S Jr, Fuster $V$ : Assessment of cardiovascular risk by use of multiplerisk-factor assessment equations. Circulation 1999; 100: 1481-1492.

13. Kodama K, Sasaki H, Shimizu Y: Trend of coronary heart disease and its relationship to risk factors in a Japanese population: a 26-year follow-up, Hiroshima/Nagasaki study. Jpn Circ J 1990; 54: 414-421.

14. Kitamura A, Iso H, Naito Y, et al: High-density lipoprotein cholesterol and premature coronary heart disease in urban Japanese men. Circulation 1994; 89: 2533-2539.

15. Fujishima F: Cardiovascular disease in elderly people: Hisayama study. Jpn J Geriatr 1999; 36: 16-21 (in Japanese).

16. Keys A, Menotti A, Aravanis C, et al: The Seven Countries Study: 2,289 deaths in 15 years. Prev Med 1984; 13: 141-154.

17. ERICA research group: Prediction of coronary heart disease in Europe: the 2 nd report of the WHO-ERICA project. Eur Heart J 1991; 12: 291-297.

18. Toshima H, Koga Y, Menotti A, et al: The Seven Countries Study in Japan. Jpn Heart J 1995; 36: 179-189.

19. Verschuren WMM, Jacobs DR, Bloemberg BP, et al: Serum total cholesterol and long-term coronary heart disease mortality in different countries: twenty-five-year follow-up of the Seven Countries Study. JAMA 1995; 274: 131-136. 